Environ. Sci. Technol., 40 (1), 74 -81, 2006.

10.1021/es050972c S0013-936X(05)00972-7

Copyright $\odot 2005$ American Chemical Society

\title{
Effects of the Antithyroid Agent Propylthiouracil in a Partial Life Cycle Assay with Zebrafish
}

\author{
Leo T. M. van der Ven,*† Evert-Jan van den Brandhof, $¥$ José H. Vos, \\ Deborah M. Power,l and Piet W. Westert
}

\begin{abstract}
Laboratory for Toxicology, Pathology, and Genetics, RIVM, TOX 12, PO Box 1, NL3720 BA Bilthoven, The Netherlands, Laboratory for Ecological Risk Assessment, RIVM, Bilthoven, The Netherlands, Expert Centre for Substances, RIVM, Bilthoven, The Netherlands, and Centro de Ciencias do Mar, Centro de Investigacao Marinha e Ambiental-Laboratorio Associado, University of Algarve, Campus de Gambelas, Faro 8005-139, Portugal

* Corresponding author phone: +31 30274 2681; fax: +31 30274 4446; e-mail:

L.van.der.ven@rivm.nl.

tLaboratory for Toxicology, Pathology and Genetics, RIVM.

fLaboratory for Ecological Risk Assessment, RIVM.

EExpert Centre for Substances, RIVM.

University of Algarve.
\end{abstract}

\begin{abstract}
:
Some ubiquitous pollutants of the aquatic environment, such as PCBs or other polyhalogenated aromatic hydrocarbons, may disrupt the thyroid hormone system. In a partial life cycle assay with zebrafish (Danio rerio), we studied the effects of the reference compound propylthiouracil (PTU) on reproduction, growth and development, histopathology of some target tissues, and plasma thyroid hormone levels. PTU induced a concentration-dependent increase of egg production with a concomitant decrease of mature oocyte size but had no effect on fertilization rate or hatching. In F1, serious dysmorphogenesis was found in 4 dph larvae at the highest PTU level tested $(100 \mathrm{mg} / \mathrm{L})$, and there was a dosedependent decrease in body length and weight at $42 \mathrm{dph}$ (significant at $100 \mathrm{mg} / \mathrm{L}$ PTU). At this time, there was also a decreased scale thickness, suggesting inhibited metamorphosis, detectable at $1 \mathrm{mg} / \mathrm{L}$ PTU and higher. PTU also induced activation of the thyroid follicles in a concentration-dependent way, in juveniles associated with hyperemia in the thyroid area, and depletion of liver glycogen. Effects in adults were associated with decreased circulating levels of the thyroid hormones $T_{3}$ and $T_{4}$. These observations indicate that disruption of the thyroid hormone system may affect the fitness of these aquatic organisms. The zebrafish model may contribute to the identification of thyroid hormone disrupting activity in water samples and also in the interpretation of histological observations in free-ranging fish species.
\end{abstract}

\section{Introduction}

Endocrine disrupting effects of environmental pollutants are of major scientific and social concern. Endocrine-active compounds encompass natural and synthetic hormones, such as $17^{3}$-estradiol and $17 \alpha$-ethynylestradiol, or industrial chemicals that interfere by stimulating or inhibiting binding or synthesis of hormones or their receptors and binding proteins. Examples of this class are pesticides and plasticizers having estrogenic activity (1). Disruption of hormonal systems in 
Environ. Sci. Technol., 40 (1), 74 -81, 2006.

vertebrates may lead to decreased fitness of the individual or its progeny and, thus, affect fitness of populations. Decreasing populations of fish or amphibians have been reported in various European countries, as well as in confined biotopes in the Americas and in South Africa $(1,2)$. In all these cases, endocrine-active compounds were the suspected cause.

The major focus of current research in this area is on compounds with estrogen agonist activity. Some reports also analyzed xeno-androgenic activity in the environment, e.g., refs 3-5. Studies on disruption of the thyroid system in fish are limited to occasional reports of incidental effects observed in the field $(6,7)$, whereas there are many environmental contaminants which can potentially disrupt the thyroid hormone system (8). Only some of these were involved in effect studies, notably some PCBs or other polyhalogenated aromatic hydrocarbons (9-12). Particular concern was raised about water-soluble goitrogens such as perchlorates that can easily contaminate drinking water (13). More recently, there is a growing interest in the potential hazard of high production volume brominated flame retardants, which are recovered from many compartments in the aquatic environment (14-16) and some of which are suspected to interact with the thyroid hormone system at environmentally relevant levels (17).

This work was part of the project aiming at the develop ment of an in vivo model to identify endocrine disrupting activity using zebrafish (Danio rerio) in a partial life cycle assay. In this paper we report the effects of a thyroid inhibitor, which was one of several reference compounds tested in the project, on the general condition of the fish, on reproduction, on histopathology, and on thyroid hormone levels. Propylthiouracil (PTU), although not a particular environmental contaminant, was chosen as a suitable reference agent because it is a powerful antithyroid drug of which the mechanism of action is well-known: it inhibits intrathyroidal synthesis of thyroid hormones by interfering with thyroid peroxidase mediated iodine utilization and the coupling of mono- and diiodotyrosines (MIT and DIT) which are required for the formation of $T_{3}$ and $T_{4}$. Furthermore, it blocks the peripheral conversion of $T_{4}$ to $T_{3}(18)$. The net effect thus is a reduced level of active thyroid hormones in peripheral tissues and feedback stimulation of the thyroid. Actions of thyroid hormones (THs) with specific relevance to the studied model are their role in early development, metamorphosis and metabolism (19). In this study, indicative end points of these aspects were investigated, in addition to general and reproductive parameters. Specifically, histopathology of thyroid follicles combined with analysis of plasma thyroid hormone levels should be indicative of thyroid axis disruption. Considering the mechanisms governing homeostasis of the endocrine system, it was anticipated that inhibition of TH synthesis by PTU would increase secretion of thyroid stimulating hormone (TSH) by the pituitary, which in turn would induce activation of the thyroid epithelium. Histology of the liver was studied because of its central role in metabolism, including that of thyroid hormones, and scale development could serve as an indicator of metamorphosis. The integration of the analyses should provide a specific pattern for antithyroid effects.

\section{Materials and Methods}

In Vivo Exposures. A stock of $10 \mathrm{~g}$ of propylthiouracil (PTU, CAS 51-51-5, Sigma-Aldrich) was prepared in $100 \mathrm{~mL}$ of DMSO, assisted with ultrasound (Bransom sonifier B12 with ultrasonic tip, $30 \mathrm{~min}$ at maximal power) to facilitate dissolution, and stored at $4^{\circ} \mathrm{C}$. This stock was used to prepare test medium concentrations at $10 \mathrm{mg}$ of PTU/L or lower; for higher concentrations, the PTU was directly dissolved in the test medium, assisted with DMSO and sonification. 
Environ. Sci. Technol., 40 (1), 74 -81, 2006.

Final concentration of DMSO in test media was adjusted to $0.01 \%$ in all groups. Actual concentrations of PTU were $106-125 \%$ of nominal values during a $96-\mathrm{h}$ period and remained constant in time.

Exposure assays were carried out with fish of ap proximately 15 months of age from a laboratory stock. The zebrafish stock was imported Singapore culture (Ruinemans Aquarium BV, Montfort, NL) and had been maintained and propagated for more than 3 years ( $3-4$ generations) in our laboratory in apparently good health and condition.

Two range finding test were conducted using concentra tion ranges of $0.1-1000 \mu$ $\mathrm{g} / \mathrm{L}$ and 32-1000 $\mathrm{mg} / \mathrm{L}$ PTU. In these assays, duplicate pairs of adult fish and duplicate batches of hatchlings per group were exposed for 10 days. On the basis of the absence of effects in the low concentration assay and on acute mortality at $1000 \mathrm{mg} / \mathrm{L}$ PTU and with the aim to include at least one effective concentration and to have adequate survival and reproduction in at least the mid- and lowconcentration groups, the range of 1,10 , and $100 \mathrm{mg} / \mathrm{L}$ PTU was selected for further testing in a partial life cycle (PLC) assay. In this test, adults were exposed for 21 days in triplicate spawning units of two females and one male per concentration. Males and females were kept separately mainly on the basis of abdominal contour, color intensity, and behavior but joined twice a week to allow spawning. The number of spawnings/experimental unit was recorded. Eggs collected after spawning were counted and monitored for fertilization and hatching. Hatchlings resulting from incubation of one spawn of each week were further maintained and sampled after 42 days of exposure to similar test or control media as the P generation.

Experiments were conducted in demineralized water supplemented with $\mathrm{NaHCO}_{3}$ $(100 \mathrm{mg} / \mathrm{L}), \mathrm{KHCO}_{3}(20 \mathrm{mg} / \mathrm{L}), \mathrm{CaCL}_{2} \cdot 2 \mathrm{H}_{2} \mathrm{O}(200 \mathrm{mg} / \mathrm{L})$, and $\mathrm{MgSO}_{4} \cdot 7 \mathrm{H}_{2} \mathrm{O}(180$ $\mathrm{mg} / \mathrm{L}$ ) and then aerated for $24 \mathrm{~h}$ at $27 \pm 2{ }^{\circ} \mathrm{C}, \mathrm{pH}$ 7.4-8.4, and $\mathrm{O}_{2}$ 5.9-8.9 mg/L. Temperature was monitored continuously, $\mathrm{pH}$ and oxygen twice in each renewal period and nitrite when increased respiration rate was suspected. Adult fish were exposed at densities of $2 \mathrm{~L} /$ fish and juveniles at $30 \mathrm{~mL} /$ fish until $21 \mathrm{dph}$, thereafter at $60 \mathrm{~mL} /$ fish, i.e. in total medium volumes of $2 \mathrm{~L}$ for adults and 1.5-3 $L$ for juveniles. Exposure was in a semistatic system with medium renewal every 3-4 days. PTU values were monitored at $24 \mathrm{~h}$ intervals and appeared to be stable for $96 \mathrm{~h}$. To this aim, water samples were spiked with methylthiouracil as internal standard and concentrated or diluted as appropriate. Analysis was performed on an Agilent HP 1100 LC-MS. Mass fragments for PTU M/Z 171 and MTU M/Z 143 were analyzed in APCI mode. The ratio of PTU:MTU was plotted against the absolute concentration, and unknown samples were calculated using the calibration line. The method had a lower limit of detection of $<1 \mathrm{ng}$ PTU/mL and a coefficient of variation of $\pm 20 \%$. Measured values were greater than $105 \%$ nominal values, irrespective of concentration (average \pm SD of all values was $122.5 \pm 37.8 \%$, of combined samples $/ 24 \mathrm{~h}$ interval in the range $t=0-96 \mathrm{~h}(105$ $\pm 34,124 \pm 25,124 \pm 51,117 \pm 36,124 \pm 50 \%)$ and of combined samples/concentration 1,10 , and $100 \mathrm{mg} / \mathrm{L}(128 \pm 47,127 \pm 37,113 \pm 29 \%))$.

Animals were monitored daily for general health and clinical effects such as mortality, abnormal behavior and appearance. At the end of experiments, fish were euthanized in an aqueous solution of $100 \mathrm{mg} / \mathrm{L}$ tricaine methanesulfonate (MS-222, CAS RN 886-86-2, Sigma-Aldrich) and neutralized with sodium bicarbonate (2/1 MS-222, weight/weight); then their length and weight were measured and blood was collected in a heparinized glass capillary tube from the tail vessels by cutting off the tail, for analysis of thyroid hormones. Animals were then fixed in toto for histopathology as described below. The Institute's Animal 
Environ. Sci. Technol., 40 (1), 74 -81, 2006.

Experiment Com mittee, according to Dutch legislation, had approved the experiments.

Radioimmunoassay for $\mathbf{T}_{\mathbf{3}}$ and $\mathbf{T}_{\mathbf{4}}$. Plasma samples were resuspended in assay buffer to the original volume when exsiccation of the relative small volumes of plasma had occurred. This had happened randomly in samples of all exposure groups. Sample heat pretreatment was carried out to eliminate nonspecific $\mathrm{TH}$ binding activity, which was apparent in zebrafish plasma. This plasma binding activity may arise from several factors which include specific TH-binding proteins (e.g. transthyretin, thyroglobulin, and albumin) or alternatively nonspecific matrix effects. A pilot assay showed that addition of aprotinin (routinely added to prevent proteolysis) had no effect on the TH assay. Competitive radioimmunoassays were used to measure thyroxine $\left(T_{4}\right)$ and triiodothyronine $\left(T_{3}\right)$. Assays for $T_{3}$ and $T_{4}$ were carried out in Tris buffer $(0.1 \mathrm{M}, \mathrm{pH} 7.5)$ or barbital buffer $(0.07 \mathrm{M}, \mathrm{pH} 8.6)$ containing $0.1 \% \mathrm{BSA}$ and $0.1 \%$ sodium azide, respectively. Standard curves were prepared with stock $T_{4}(1 \mathrm{mg} / \mathrm{mL})$ and $T_{3}(1$ $\mathrm{mg} / \mathrm{mL}$, Sigma-Aldrich, Madrid. Spain) dissolved in $0.1 \mathrm{M} \mathrm{NaOH}$. Radiotracers, ${ }^{125} \mathrm{I}_{-} \mathrm{T}_{4}$ and ${ }^{125} \mathrm{I}-\mathrm{T}_{3}$ (NEN, DuPont), were diluted for use in the appropriate assay buffer and antisera to $T_{4}$ and $T_{3}$ (Sigma-Aldrich) were used at a working dilution of $1 / 4000$ and $1 / 10000$, respectively. The total assay volume was $400 \mu \mathrm{L}$ and contained either $100 \mu \mathrm{L}$ of standard or varying volumes and dilutions of plasma, $100 \mu \mathrm{L}$ of anti- $\mathrm{T}_{4}$ or anti- $\mathrm{T}_{3}$, and $100 \mu \mathrm{L}$ of radiotracer with the exception of tubes to determine total $\mathrm{cpm}$ and nonspecific binding without antisera. Tubes were vortexed and incubated for $16-24 \mathrm{~h}$ at $4{ }^{\circ} \mathrm{C}$. Separation of the free hormone from the bound hormone was by the second antibody method.

Histology. Details on histology have been described previously (20). In brief, animals were fixed in Bouin's fixative for $24 \mathrm{~h}$, then transferred and stored in $70 \%$ ethanol, and embedded in paraffin after dehydration in a graded ethanol/xylene series. Sections of $4 \mu \mathrm{m}$ were prepared in a coronal (horizontal) plane to allow analysis of target organs, in particular thyroid tissue and scales. Sections were dewaxed, rehydrated, stained with haematoxylin and eosin, and then mounted in Tissue-Tek Resin (Sakura Finetek) and covered with a glass coverslip.

Histological assessment was done either by using qualitative descriptors or by blinded semiquantitative scoring using arbitrary intensity/severity categories of these descrip tors (see Table 2). Additionally, morphometrical quantifica tion was conducted for size of mature oocytes in the ovary of $\mathrm{P}$ females and for scale thickness in F1, essentially as described previously (20). Since development is obviously correlated to growth, animals were matched for length for the analysis of scale thickness, in two cohorts of respectively 12 and $16 \mathrm{~mm}$ animals. Liver glycogen contents were estimated by histology on the same $\mathrm{H}$ and $\mathrm{E}$ sections.

Statistics. Each spawning unit (two males, one female) in a single tank was defined as the experimental unit of the PLC tests, and the subsequent (not independent) egg deposits in such a tank and the resulting hatches were defined as repeated measures. All life history parameters were analyzed with One-Way ANOVA followed by Dunnett's Multiple Comparison to compare treatments with the controls. Correlation of effects with exposure concentration was analyzed by linear regression analysis (life history variables and other continuous end points, such as hormone concentrations and histomorphometrical data). Different treat ments of progeny from a single experimental unit were analyzed by paired $t$-tests with the adult couples defining the pairs. Distribution of histological grading was analyzed with $\boldsymbol{x}^{2}$ and Fisher's exact tests. 
Environ. Sci. Technol., 40 (1), 74 -81, 2006.

\section{Results}

In Vivo Effects. In life observations of parental fish revealed no effect on survival, appearance (notably no goiter, i.e. swelling and redness of the thyroid area), and behavior of PTU exposure compared to control fish. Linear regression analysis of total number of eggs/female (no transformation) revealed a positive correlation with PTU treatment (Table 1э). There was no significant change in number of spawnings, mean spawn size, or fertilization between control and treated groups (ANOVA), probably as a consequence of the highly variable nature of egg production among and between individuals. There was, however, a concentration-dependent decrease of the size of mature oocytes, as measured histomorphometrically in the ovary (Table 1 ).

Treatment with PTU did not cause a change in hatch rate, which had, for unknow reasons, overall low values (56\% in controls) compared to historical controls (77$100 \%)$. Oc casionally, PTU exposed larvae were malformed, although there was only a significant increase in the incidence of malformed larvae detected in the $100 \mathrm{mg} / \mathrm{L}$ PTU treatment group compared to untreated larvae $(p=0.01)$. Abnormal behavior, such as immobility, was recorded only sporadically. Juvenile survival was low (55-72\%) in all groups, including controls, compared to historical survival rates of the nonexperimental stock, which normally range approximately $75-96 \%$. There were no obvious explanations for this observation. The length of juveniles was significantly reduced by exposure to $100 \mathrm{mg} / \mathrm{L} \mathrm{PTU}$, irrespective of parental treatment. There was also a significant concentrationdependent reduction in both length and weight after exposure of only parents and after exposure of both parents and offspring. As a consequence of the concomitant reduction in length and weight, the condition factor was not significantly altered from controls by PTU treatment. From $28 \mathrm{dph}$ onward, swelling and hyperemia of the thyroid area (goiter), as well as reduced pigmentation, was observed with varying severity in the high dose PTU groups, compared to the other groups (Figure 1a). 

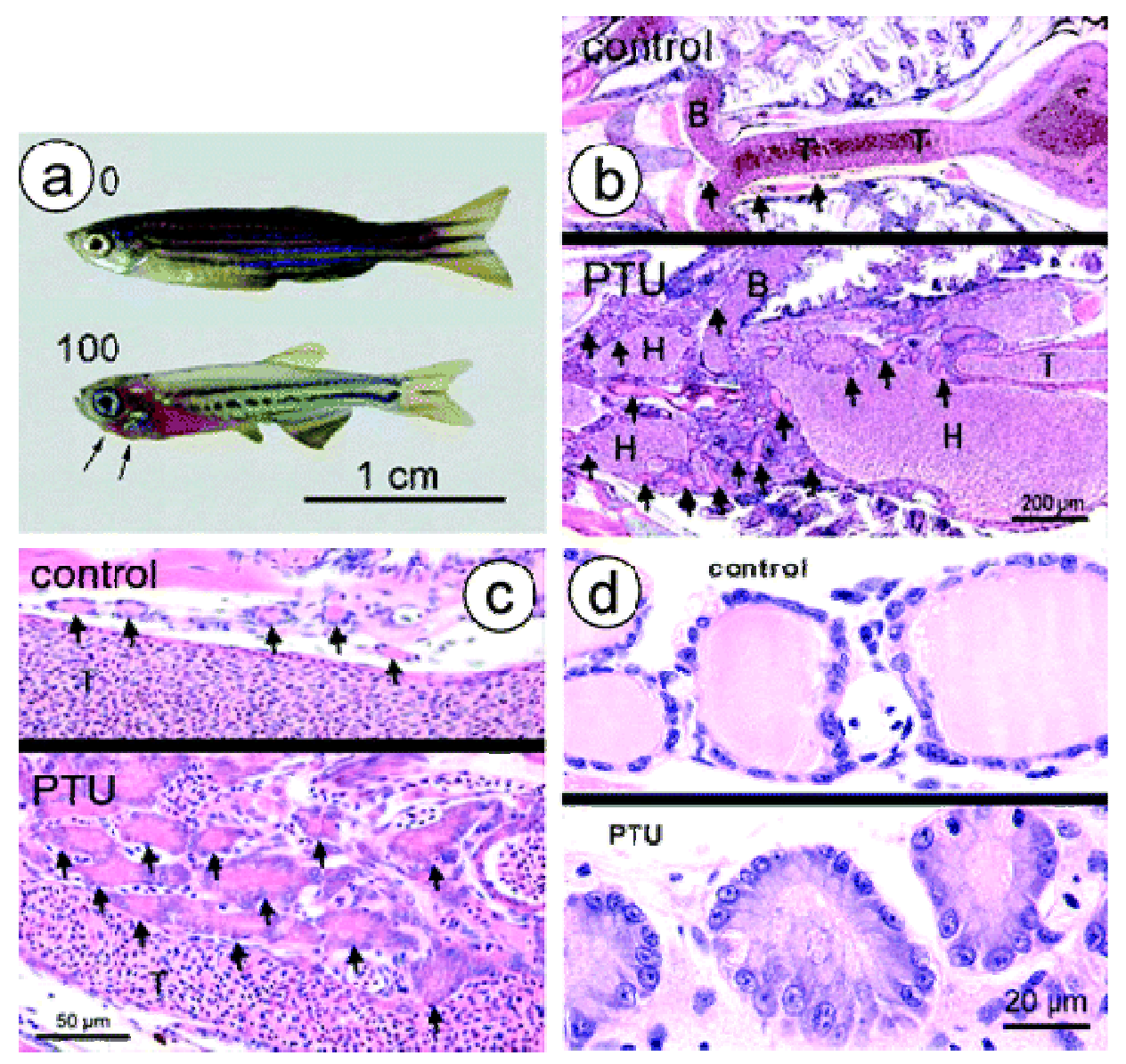

Figure 1 Effects on the thyroid gland (area): (a) Goiter and hyperemia (arrows) and poor pigmentation in 42-dph juvenile F1 zebrafish after treatment with 100 $\mathrm{mg} / \mathrm{L} \mathrm{PTU;} \mathrm{(b,} \mathrm{c)} \mathrm{low} \mathrm{and} \mathrm{intermediate} \mathrm{power} \mathrm{microphotograph} \mathrm{of} \mathrm{the} \mathrm{jugular} \mathrm{area}$ of juvenile $\mathrm{F} 1$ zebrafish (42 dph), control (top) and after exposure to $100 \mathrm{mg} / \mathrm{L}$ PTU, showing numerous activated thyroid follicles (arrows) and extensive hyperaemia $(\mathrm{H})$ after exposure to PTU $(\mathrm{T}$, truncus arteriosus; $\mathrm{B}$, branchial arteries); (d) high-power thyroid histology in adult zebrafish. In the control, follicles are well filled with colloid and follicle cells are cuboidal (top); after exposure to $320 \mathrm{mg} / \mathrm{L}$ PTU for 10 days (bottom), follicles are depleted and the epithelium has a columnar morphology.

Histopathology: Thyroid. In control animals, thyroid follicles were found dispersed in the loose connective tissue adjacent to the ventral aorta and its final rostral branching. Most follicles were small, well filled with colloid, and had lowcuboid or flat epithelium; one relatively large follicle was invariably present directly rostral to the aorta branching. At $42 \mathrm{dph}$, surviving juveniles exposed to 10-100 mg/L PTU showed histological signs of goiter such as hyperplasia and hypertrophy of the thyroid follicles, comparable to the picture in adults (see Figure $1 \mathrm{~b}-\mathrm{d}$ ). Usually the activated follicles were small, although occasionally macrofollicular struma was seen. The increased volume of the thyroid tissue caused expansion of the interbranchial tissue compartment with extension into the adjacent gills and along the jugular vein (Figure 1b,c). Exposure to PTU also caused activation of the thyroid follicular cells (Figure 1d, bottom), indicated by the columnar appearance of follicle epithelium (compare with the reference thyroid follicles in Figure 1d, top), hypochromasy, and increase of size of the nuclei and by the basophilic cytoplasm. In addition, depletion of colloid 
Environ. Sci. Technol., 40 (1), 74 -81, 2006.

(thyroglobulin) was evident. The observed thyroid activation was PTU concentration dependent (Table 20 ) and time dependent, since the effects appeared more intense in the 21-day test compared to the 10-day exposure pilot (data not shown). This table also indicates that there was an effect of parental exposure (observed effects after exposure of parental animals only or more severe effects after consecutive parental and juvenile exposure compared to exposure of juveniles only). Furthermore, this thyroid pathology appeared less severe in adults than in the F1 juveniles.

There was also a striking hyperemia in this region, as seen by marked and tortuous dilation of the jugular vein, the interfollicular capillaries, and the branchial vessels (Figure 1b). Occasionally, edema was observed in the secondary gill lamellae, the latter probably due to physical circulatory insufficiency resulting from the struma.

Thyroid Hormones. Analysis of thyroid hormone in blood plasma of these adult fish showed that in control zebrafish circulating levels of $T_{4}$ were higher than $T_{3}$. Moreover, PTU treatment significantly decreased circulating $T_{4}$ at both 10 and 100 $\mathrm{mg} / \mathrm{L}$ and $\mathrm{T}_{3}$ was significantly reduced only at the latter concentration (Figure 2 ). There was also a significant concentration-dependent decrease of both hormones.

\section{thyroid hormone levels in blood plasma after PTU}

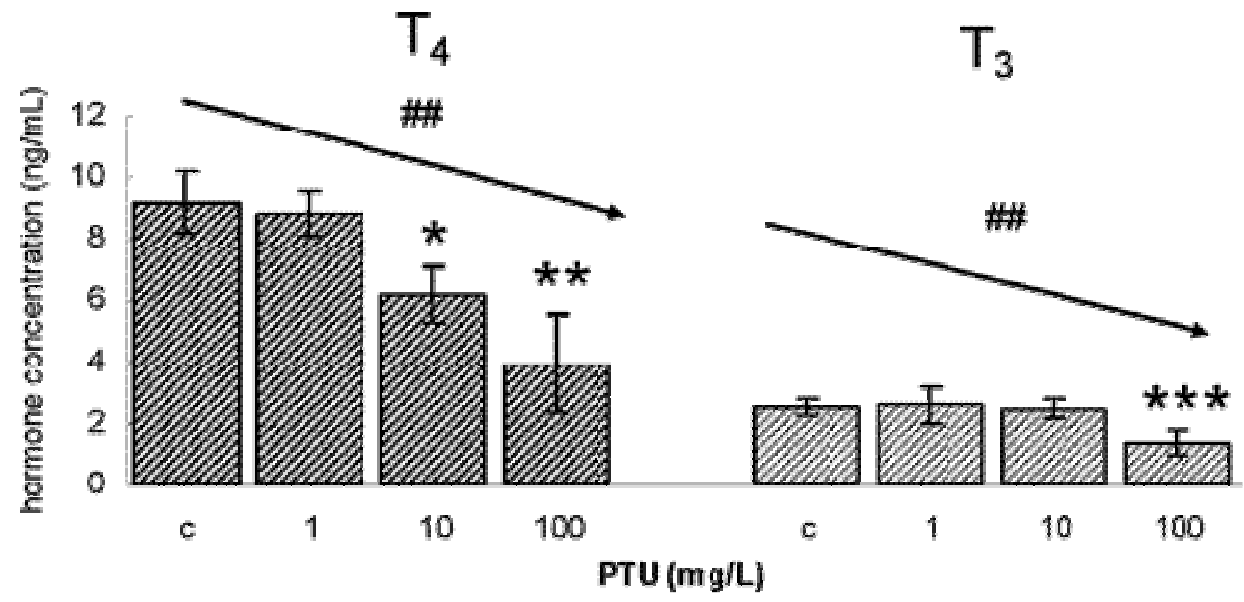

Figure 2 Thyroid hormone analysis following exposure to PTU. $\mathrm{T}_{4}$ is decreased at 10 and $100 \mathrm{mg} / \mathrm{L}$ PTU ( $p<0.05$ and $p<0.01$, respectively). There is a concentration-dependent decrease (arrow and \#\#, regression analysis, $p=$ $\left.0.0005, r^{2}=0.5709\right)$. $T_{3}$ is decreased at $100 \mathrm{mg} / \mathrm{L}$ PTU ( $T$-test, $p<0.0001$ ). Linear regression analysis revealed also a concentration-dependent decrease (arrow and \#\#, $p<0.0001, r^{2}=0.5734$ ).

Histopathology: Liver. A concomitant effect was depletion of liver glycogen from $1 \mathrm{mg} / \mathrm{L}$ onward, assessed as unstained cytoplasmic areas with a flocculent aspect, morphologically distinct from lipid, which normally presents as globular sharply delineated vacuoles (Figure 3 ) and which was PTU concentrationdependent in severity. There appeared to be a transgenerational effect of parental exposure on liver glycogen depletion. 

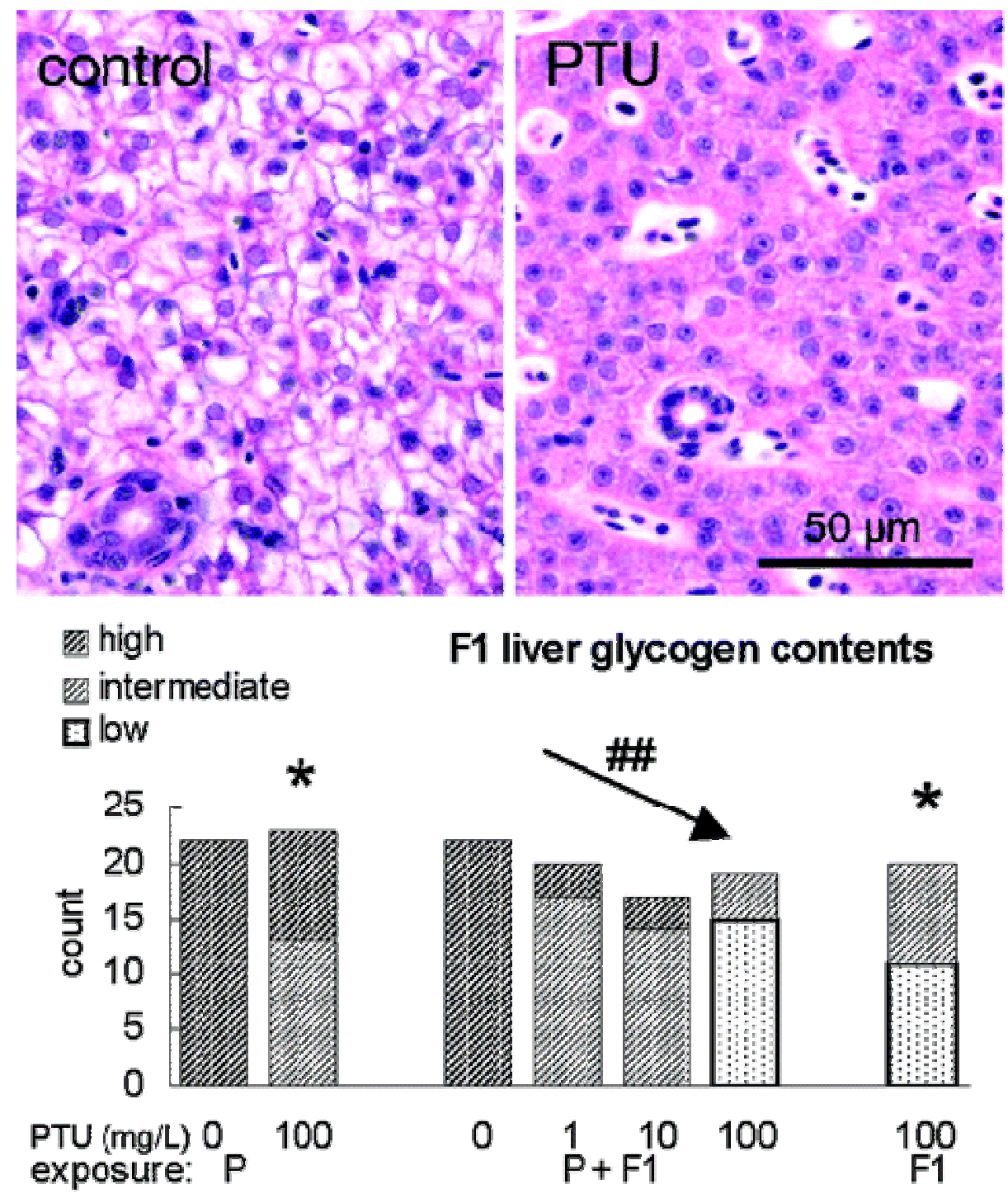

Figure 3 Liver glycogen storage after PTU in F1 zebrafish And liver histology of juvenile zebrafish after 42 days of exposure to PTU. The histology of the liver of the control fish (left) shows high glycogen contents (see text). After exposure to $100 \mathrm{mg} / \mathrm{L}$ PTU (right microphotograph), the liver is virtually depleted of glycogen. Categorization of hepatocyte glycogen contents by histological estimation in 16$23 \mathrm{~F} 1$ animals/group shows that there is a significant association between activation intensity and PTU concentration for groups with same treatment for $\mathrm{P}$ and $\mathrm{F} 1$ (middle set of bars; arrow and \#\#, $p<0.0001, x^{2}=107.37$ ). There is also increased glycogen depletion after parental only (slight) or juvenile exposure only (severe; $p<0.01$; Fisher's exact test when compared with control animals).

Histopathology: Scales. In this PLC, the thickness of the scale plates was selected as a parameter for histological evaluation indicative of metamorphosis (Figure 4). Exposure to $10 \mathrm{mg}$ PTU/L and higher in groups of $12 \mathrm{~mm}$ sized animals and to $1 \mathrm{mg} \mathrm{PTU} / \mathrm{L}$ and higher in groups of $16 \mathrm{~mm}$ sized fish yielded significant inhibition of normal scale development, and the severity of the effect was concentration dependent. Exposure of only F1 (group c-100 in Figure 4) induced an effect similar to that for continuous P and F1 exposure (group 100- 
100 in Figure 4), in the case of $100 \mathrm{mg} \mathrm{PTU/L}$ (no lower dosage groups only F1 exposed were available).

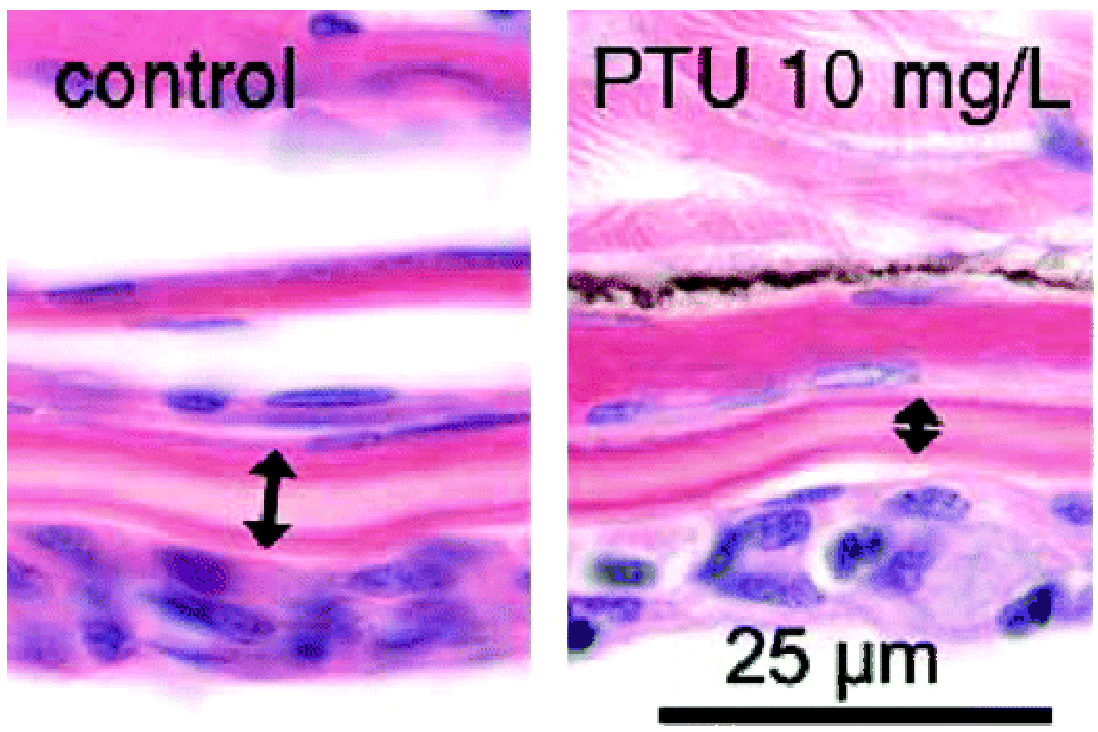

scale thickness in juvenile zebrafi

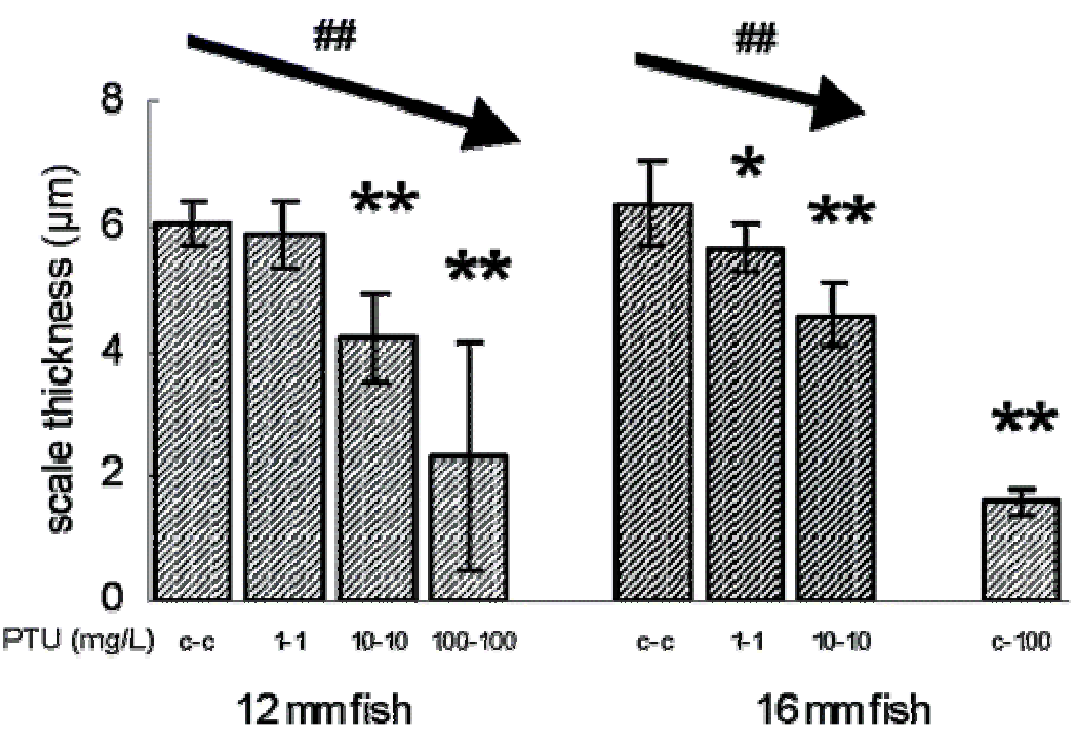

Figure 4 Decreased scale thickness after exposure to PTU of juvenile zebrafish (42 $\mathrm{dph}$ ). Microphotographs of the integument show that the scale thickness is decreased after PTU exposure (10 mg/L PTU); compare arrows in the left (control) and right (exposed) images. Lining epithelium is at the bottom, and subcutaneous tissue on top. Morphometrical measurements confirm that scale thickness is decreased after exposure to PTU in two cohorts, of 12 (light shading) and $16 \mathrm{~mm}$ (dark shading) juvenile zebrafish, respectively. Key: */**, statistically different from respective control in a Student $T$-test; arrow and \#\#, $p$ $<0.0001$ concentration-dependent effect (regression analysis). Group designations represent $\mathrm{P}$ and $\mathrm{F} 1$ exposures, respectively. Six scales were measured at a comparable level/fish in each treatment group; each bar represents the average of six fish, which, if possible, were sampled from different replicate groups. 
Environ. Sci. Technol., 40 (1), 74 -81, 2006.

There were no other PTU-related effects, notably no effect on sex ratio and morphology of thymus and adrenal cells. Compared to historical controls, a high incidence of other pathologies, (focal) peritonitis and bile duct hyperplasia, was recorded but was considered unrelated to PTU treatment since these were also observed in unexposed animals.

\section{Discussion}

TH and Energy Balance. Changes in some of the analyzed end points in this study are associated with redistribution of energy resources. Hepatic glycogen depletion, which was PTU concentration dependent, is in line with the stimulating effect of thyroid hormone on glycogen synthesis, as observed in rat hepatocytes (21), assuming that, conversely, decreased TH levels will lead to decreased glycogen synthesis. This effect, together with the reduced growth in juveniles, suggests reduced availability or increased utilization (waste) of energy. Remarkably, these effects in juveniles were also induced by only parental exposure with a high concentration of PTU; this transgenerational effect may be due to a reduced transfer of THs into the egg, thus limiting the available TH for the fish embryo, which depends on maternal THs during its early development (22). This concept of balanced TH concentra tions in the maternal plasma/ovarian fluid and the developing oocyte comes from observations in medaka and rainbow trout that $T_{3}$ diffuses freely across the egg membrane $(23,24)$. A redistribution of energy (increased utilization) is also suggested by the increased egg production in parental females. This is, however, probably a relative change, since it may be counterbalanced by decreased egg size, as suggested from measurement of mature eggs in the ovary. Such a tradeoff is known to occur under various conditions (reviewed in ref 25) and is considered a disadvantage for the developing embryo because of decreased nourishment supply; this could then in turn be an additional cause for the delayed develop ment of F1 animals.

Thyroid Hormone System. Propylthiouracil inhibits the synthesis of thyroid hormones at several points in the synthesis pathway; it interferes with thyroid iodine utilization and it inhibits peripheral conversion of $T_{4}$ to $T_{3}(18)$. The decreased levels of $T_{3}$ and $T_{4}$ hormones in adult zebrafish confirmed this inhibiting effect, which was also observed in developing zebrafish embryos, although at a higher concentration, in a short-term study (26). Histopathological observations in our fish indicated concomitant thyroid follicular cell activation in a concentration-dependent way, as evidenced by increase in cell size (columnar) and hypertrophy of the synthetic apparatus (RER, Golgi complex, and mitochondria), as seen with strong basophilia. This indicates increased (protein) synthetic activity, likely a result of feedback-induced secretion of thyrotropic hormone (TSH) by the pituitary. These observations are in line with observed effects in mammals (27).

Thyroid activation is an attempt to compensate for reduced circulating hormone levels, but it must be regarded relatively ineffective since it was observed at the same exposure level (10 mg PTU/L) that induced the thyroid hormone decrease. Furthermore, the lower PTU level in this experiment does apparently not need to be counteracted and may therefore be considered as not effective. Since thyroid follicular cell activation in juveniles occurred at the same PTU exposure range as in adults, the effective PTU level for disruption of the TH system can be considered similar in both age classes. However, the stronger thyroid activation in juveniles suggests that the compensating stimulation of thyroid hormone synthesis is more effective in adults. Alternatively, this may simply be due to the 
Environ. Sci. Technol., 40 (1), 74 -81, 2006.

different duration of the exposure ( 21 days for $\mathrm{P}$ animals, 42 days for $\mathrm{F} 1$ animals), including a lower initial parental TH supply.

A further difference between effects of PTU in adults and juveniles was the extensive hyperemia in thyroid area in the latter. Assuming similar mechanisms in zebrafish and rats, the observed hypervascularity in our zebrafish might be analogous to increased blood flow in rats; this was suggested to be an effect of increased TSH, in combination with PTU-increased sensitivity of the vascular conductance in the thyroid to TSH (28) rather than the secondary result of an increased circulatory demand or an obstructive mechanism. Similar hyperemia, interpreted as angiogenesis, was observed by Patiño et al. in zebrafish after exposure to ammonium perchlorate, and it was found to be associated with the progression of the thyroid hyperplasia to neoplastic lesions (adenoma or carcinoma-like), similar to observations in mammals (29). Both the thyroid hyperplasia and the vascular dilatation likely have caused the swelling and red coloration observed grossly in the mandibular area.

As with thyroid activation, the different outcome for hyperaemia between the adult and juvenile cohorts can possibly be explained by the different duration of the exposure.

Reduced Scale Development (Metamorphosis). In com mon with other lower vertebrates, teleosts pass through a stage of metamorphosis, which marks the transition of the larval stage to the juvenile stage. In flatfish, such as flounder, this is apparent by the obvious changes in body architecture. In zebrafish, some characteristics of this metamorphosis are the outgrowth and development of paired fins (pectoral and pelvic), the appearance of the adult pigmentation pattern (stripes), and the development of scales (19). Metamorphosis is controlled by thyroid hormones (reviewed in ref 22), and therefore, goitrogens may be expected to affect the mentioned features. In this PLC, the thickness of the scale plates was selected as a histological endpoint, and it proved to be significantly reduced upon exposure to PTU (Figure 4). The animals were matched for length to prevent bias from growth, which is better correlated to development than age. Other studies with goitrogens in fish showed effects on fin development in zebrafish (19), change in body morphology in summer flounder (30), and cartilage development in dogfish (31). These findings support the specificity of the effects on scale thickness observed in our assay and its representativity for metamorphosis, and it is further sup ported by the reduced pigmentation in the $100 \mathrm{mg}$ PTU/L group. This inhibitory effect of PTU may reflect just a delay of morphogenesis with no functional implications. On the other hand, associated changes such as delayed development of the intestinal tract (19) (not specifically studied in this experiment) may imply impaired fitness of the exposed animals.

None of the other analyzed parameters showed changes at $1 \mathrm{mg}$ PTU/L. The marginal though statistically significant decrease of scale thickness in large class juvenile fish at this low concentration suggests that scale development per se may be much more sensitive to PTU treatment than its direct effect on circulating $\mathrm{TH}$ and thyroid activation. This may be explained by the action of PTU, even at the lower concentration, at the target tissue level (inhibition of $\mathrm{T}_{4}$ conversion by deionidase), or by mitigation of the $\mathrm{TH}$ surge which is associated with the onset of metamorphosis, as observed in some species (22). A similar sensitivity was observed in a short-term morphological assay with PTU, which showed that tail resorption, a marker for metamorphosis, was inhibited in Xenopus leavis at tested exposure concentration of $2 \mathrm{mg} / \mathrm{L}$ (32). Remarkably, the sensitivity of metamorphosis appeared to be lower in a recently proposed similar assay with standardized staging of metamorphosis in Xenopus laevis tadpoles for monitoring 
Environ. Sci. Technol., 40 (1), 74 -81, 2006.

thyroid hormone system disrupting effects by two independent groups. Opitz et al. showed that, in the Xenopus assay, significant inhibition of development can be detected with $25 \mathrm{mg} / \mathrm{L}$ ethylenethiourea (ETU), which was 2-4-fold lower than the ETU concentrations inducing a comparable effect to their PTU reference concentrations of $75-100 \mathrm{mg} / \mathrm{L}$ (33); in the study of Degitz et al., the lowest effect concentration for development was $10-20 \mathrm{mg} / \mathrm{L}$ PTU (34). Elsalini et al. proposed a zebrafish larvae model as an alternative for the amphibian metamorphosis assay, by evaluating the in situ expression of $\mathrm{T}_{4}$ intake in thyroid follicles (26). Our results with metamorphosis inhibition in this species confirm that the zebrafish is a sensitive model for hazard identification with compounds acting on the thyroid hormone system.

Overall Hazard. This study did not indicate a direct threat to life or reproduction of the goitrogen PTU at the concentrations tested in this study, since there was no decreased survival, fertilization rate, or hatching under these low-stress laboratory conditions. Nevertheless, the sum of adverse effects related to metabolism and development, notably in progeny, suggests a decreased overall fitness, which could easily reduce homeostasis, thus increasing vulnerability to other stressors. In addition, Nigrelli et al. discussed adverse secondary effects of goiter/thyroid hyperplasia and neoplasia on respiration and kidney function in the species they studied, Pacific coral catfish and Atlantic yellowhead jawfish (35). Although PTU itself is not necessarily an environmental pollutant itself, the results with this reference compound indicate that environmental contaminants with goitrogenic activity, such as reviewed by Kime (8), or, of more recent interest, certain brominated flame retardants (17), may contribute to impairment of the overall condition of aquatic organisms and their populations, the greater when combinatorial effects of compounds with direct and indirect activity on the thyroid and its hormones or on other endocrine systems are considered.

In conclusion, in zebrafish impaired thyroid hormone synthesis induced by PTU leads to detectable thyroid hypertrophy from $1 \mathrm{mg} / \mathrm{L}$ (marginal) onward. Depletion of liver glycogen is likely a manifestation of the effect of the hypothyroidy on metabolism, as is the suggested shifted balance between egg volume and egg production, and retarded scale development may be a manifestation of inhibited metamorphosis. This indeed represents a coherent set of changes, for the most part specific for disruption of the thyroid hormone system. Specificity is further supported by the correlation of severity of these effects with exposure concentration. Through these effects, goitrogens may imply a serious hazard for aquatic organisms and populations. The PLC with zebrafish is an effective tool to monitor for such effects of (potential) environmental contaminants and can thus contribute to hazard identification and risk assessment of these substances.

\section{Acknowledgment}

Piet Beekhof, Bhawani Nagarajah, Sisca de Vlugt, Joke Robinson, and Sandra de Waal are acknowledged for their dedicated technical assistance, and Dr. Saskia Sterk (Labora tory for Food and Residue analysis, European Union Com munity Reference Laboratory, RIVM) is acknowledged for PTU measurements. This study was ordered by the European Commission (Project B6-7920/98/00025) and the Dutch Environment Ministry VROM (Project M/640920). 
Environ. Sci. Technol., 40 (1), 74 -81, 2006.

\section{References}

1. Tyler, C. R.; Jobling, S.; Sumpter, J. P. Endocrine disruption in wildlife: a critical review of the evidence. Crit. Rev. Toxicol. 1998, 28, 319-361.

2. Vos, J. G.; Dybing, E.; Greim, H. A.; Ladefoged, O.; Lambre, C.; Tarazona, J. V.; Brandt, I.; Vethaak, A. D. Health effects of endocrine-disrupting chemicals on wildlife, with special refer ence to the European situation. Crit. Rev. Toxicol. 2000, 30, 71-133.

3. Baatrup, E.; Junge, M. Antiandrogenic pesticides disrupt sexual characteristics in the adult male guppy Poecilia reticulata. Environ. Health Perspect. 2001, 109, 1063-1070.

4. Thomas, K. V.; Hurst, M. R.; Matthiessen, P.; McHugh, M.; Smith, A.; Waldock, M. J. An assessment of in vitro androgenic activity and the identification of environmental androgens in United Kingdom estuaries. Environ. Toxicol. Chem. 2002, 21, 1456-1461.

5. Ellis, R. J.; van den Heuvel, M. R.; Bandelj, E.; Smith, M. A.; McCarthy, L. H.; Stuthridge, T. R.; Dietrich, D. R. In vivo and in vitro assessment of the androgenic potential of a pulp and paper mill effluent. Environ. Toxicol. Chem. 2003, 22, 1448-1456.

6. Moccia, R. D.; Leatherland, J. F.; Sonstegard, R. A. Increasing frequency of thyroid goiters in Coho salmon (Oncorhynchus kisutch) in the Great Lakes. Science 1977, 198, 425-426.

7. Moccia, R. D.; Leatherland, J. F.; Sonstegard, R. A. Quantitative interlake comparison of thyroid pathology in Great Lakes coho (Oncorhynchus kisutch) and chinook (Oncorhynchus tschawytscha) salmon. Cancer Res. 1981, 41, 2200-2210.

8. Kime, D. E. In Endocrine disruption in fish; Kluwer Academic Publishers: Boston, Dordrecht, London, 1998; Chapter 10.

9. Ram, R. N. Carbofuran-induced histophysiological changes in thyroid of the teleost fish, Channa punctatus (Bloch). Ecotoxicol. Environ. Saf. 1988, 16, 106-113.

10. Kirubagaran, R.; Joy, K. P. Toxic effects of mercurials on thyroid function of the catfish, Clarias batrachus (L.). Ecotoxicol. Environ. Saf. 1989, 17, 265-271.

11. Ram, R. N.; Sathyanesan, A. G. Histopathological changes in liver and thyroid of the teleost fish, Channa punctatus (Bloch), in response to ammonium sulfate fertilizer treatment. Ecotoxicol. Environ. Saf. 1987, 13, 185-190.

12. Brouwer, A.; Morse, D. C.; Lans, M. C.; Schuur, A. G.; Murk, A. J.; Klasson-Wehler, E.; Bergman, A.; Visser, T. J. Interactions of persistent environmental organohalogens and the thyroid hormone system: Mechanisms and possible consequences for animal and human health. Toxicol. Ind. Health. $1998,14,59-84$.

13. Brechner, R. J.; Parkhurst, G. D.; Humble, W. O.; Brown, M. B.; Herman, W. H. Ammonium perchlorate contamination of Colorado River drinking water is associated with abnormal thyroid function in newborns in Arizona. J. Occup. Environ. Med. 2000, 42, 777-782.

14. de Wit, C. A. An overview of brominated flame retardants in the environment. Chemosphere 2002, $46,583-624$.

15. Sellstrom, U.; Bignert, A.; Kierkegaard, A.; Haggberg, L.; de Wit, C. A.; Olsson, M.; Jansson, B. Temporal trend studies on tetra- and pentabrominated diphenyl ethers and hexabromocyclododecane in guillemot egg from the Baltic Sea. Environ. Sci. Technol. 2003, 37, 5496-5501.

16. Morris, S.; Allchin, C. R.; Zegers, B. N.; Haftka, J. J.; Boon, J. P.; Belpaire, C.; Leonards, P. E.; Van Leeuwen, S. P.; de Boer, J. Distribution and fate of HBCD and TBBPA brominated flame retardants in North Sea estuaries and aquatic food webs. Environ. Sci. Technol. 2004, 38, 5497-5504.

17. Legler, J.; Brouwer, A. Are brominated flame retardants endocrine disruptors? Environ. Int. 2003, $29,879-885$.

18. Cooper, D. S. Antithyroid drugs. N. Engl. J. Med. 2005, 352, 905-917.

19. Brown, D. D. The role of thyroid hormone in zebrafish and axolotl development. Proc. Natl. Acad. Sci. U.S.A. 1997, 94, 13011-13016.

20. Van der Ven, L. T. M.; Wester, P. W.; Vos, J. G. Histopathology as a tool for the evaluation of endocrine disruption in zebrafish. Environ. Toxicol. Chem. 2003, 22, 908-913.

21. Bollen, M.; Stalmans, W. The effect of the thyroid status on the activation of glycogen synthase in liver cells. Endocrinology 1988, 122, 2915-2919. 
Environ. Sci. Technol., 40 (1), 74 -81, 2006.

22. Power, D. M.; Llewellyn, L.; Faustino, M.; Nowell, M. A.; Bjornsson, B. T.; Einarsdottir, I. E.; Canario, A. V.; Sweeney, G. E. Thyroid hormones in growth and development of fish. Comp. Biochem. Physiol., Part C: Toxicol. Pharmacol. 2001, 130, 447-459.

23. Tagawa, M.; Brown, C. L. Entry of thyroid hormones into tilapia oocytes. Comp. Biochem. Physiol., Part B: Biochem. Mol. Biol. 2001, 129, 605-611.

24. 24. RAINE, J. C.; LEATHERLAND, J. F. TRAFFICKING OF L-triiodothyronine between ovarian fluid and oocytes of rainbow trout (Oncorhynchus mykiss). Comp. Biochem. Physiol., Part B: Biochem. Mol. Biol. 2003, 136, 267-274.

25. Kime, D. E. In Endocrine disruption in fish; Kluwer Academic Publishers: Boston, Dordrecht, London, 1998; Chapter 9.

26. Elsalini, O. A.; Rohr, K. B. Phenylthiourea disrupts thyroid function in developing zebrafish. Dev. Genes Evol. 2003, 212, 593-598.

27. Yamasaki, K.; Tago, Y.; Nagai, K.; Sawaki, M.; Noda, S.; Takatsuki, M. Comparison of toxicity studies based on the draft protocol for the 'Enhanced OECD Test Guideline no. 407' and the research protocol of 'Pubertal Development and Thyroid Function in Immature Male Rats' with 6-n-propyl-2thiouracil. Arch. Toxicol. 2002, 76, 495-501.

28. Connors, J. M.; Huffman, L. J.; Michalkiewicz, M.; Chang, B. S.; Dey, R. D.; Hedge, G. A. Thyroid vascular conductance: differential effects of elevated plasma thyrotropin (TSH) induced by treatment with thioamides or TSH-releasing hormone. Endocrinology 1991, 129, 117-125.

29. Patiño, R.; Wainscott, M. R.; Cruz-Li, E. I.; Balakrishnan, S.; McMurry, C.; Blazer, V. S.; Anderson, T. A. Effects of ammonium perchlorate on the reproductive performance and thyroid follicle histology of zebrafish. Environ. Toxicol. Chem. 2003, 22, 1115-1121.

30. Schreiber, A. M.; Specker, J. L. Metamorphosis in the summer flounder (Paralichthys dentatus): stage-specific developmental response to altered thyroid status. Gen. Comp. Endocrinol. 1998, 111, 156-166.

31. Alluchon-Gerard, M. J. Influence of thyroidectomy and PTU treatment on cartilage ultrastructure in the embryo and very young dogfish (scyllium canicula, chondrichthyes). Arch. Anat. Microsc. Morphol. Exp. 1982, 71, 51-70.

32. Fort, D. J.; Rogers, R. L.; Morgan, L. A.; Miller, M. F.; Clark, P. A.; White, J. A.; Paul, R. R.; Stover, E. L. Preliminary validation of a short-term morphological assay to evaluate adverse effects on amphibian metamorphosis and thyroid function using Xenopus laevis. J. Appl. Toxicol. 2000, 20, 419425.

33. Opitz, R.; Braunbeck, T.; Bogi, C.; Pickford, D. B.; Nentwig, G.; Oehlmann, J.; Tooi, O.; Lutz, I.; Kloas, W. Description and initial evaluation of a Xenopus metamorphosis assay for detection of thyroid system-disrupting activities of environmental compounds. Environ. Toxicol. Chem. 2005, 24, 653-664.

34. Degitz, S. J.; Holcombe, G. W.; Flynn, K. M.; Kosian, P. A.; Korte, J. J.; Tietge, J. E. Progress towards development of an amphibian-based thyroid screening assay using Xenopus laevis. Organismal and thyroidal responses to the model compounds 6-propylthiouracil, methimazole, and thyroxine. Toxicol. Sci. 2005, 87, 353-364.

35. Nigrelli, R. F.; Ruggieri, G. D. Hyperplasia and neoplasia of the thyroid in marine fishes. Mt. Sinai J. Med. 1974, 41, 283-293. 
Table 1. Effect of PTU on in Vivo End Points: Reproduction and Development ${ }^{a}$

$P$, reproductn

exposed PTU

generatn $(\mathrm{mg} / \mathrm{L})$

P $\quad 0$

1

100

concn-

dependt ns

change

$P+F 1 \quad 0$

1

10

100

concn-

dependt

change

F1 100
F1, developmt and growth

no. of spawn tot. oocyte fertilizatn hatching length malformatn body survival conditn

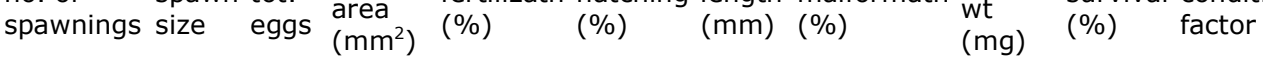

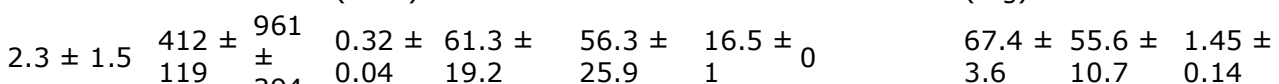

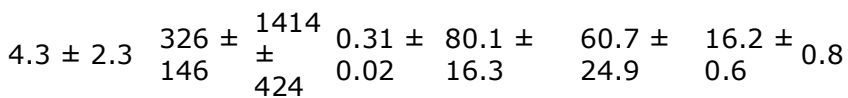

$62.7 \pm 67.4 \pm 1.41 \pm$

$\begin{array}{lll}5.2 & 16.1 & 0.09\end{array}$

$\begin{array}{lllllll}4.7 \pm 1.2 & 299 \pm & \begin{array}{l}1394 \\ \pm\end{array} & 0.26 \pm & 74.9 \pm & 74.9 \pm & 15.6 \pm \\ & 202 & 335 & 0.02 & 19.8 & 24.8 & 0.3\end{array}$

$61.5 \pm 64.9 \pm 1.59 \pm$

$\begin{array}{lll}1.4 & 21.7 & 0.05\end{array}$

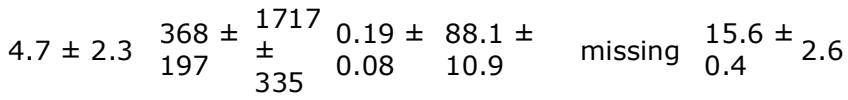

$57.4^{d} \pm 59.7 \pm 1.44 \pm$

$\begin{array}{lll}6.4 & 4.5 & 0.02\end{array}$

ns $\quad b \quad$ ns $\quad b \quad$ ns ns

$56.3 \pm 16.5 \pm 0 \quad 67.4 \pm 55.6 \pm 1.45 \pm$

$\begin{array}{llllll}25.9 & 1 & 0 & 3.6 & 10.7 & 0.14\end{array}$

$61.1 \pm 16.4 \pm 0.5 \quad 66.2 \pm 72.2 \pm 1.42 \pm$

$\begin{array}{llllll}22.7 & 0.5 & 0.5 & 10.5 & 22.5 & 0.1\end{array}$

$78.4 \pm 16 \pm 3.4 \quad 62.7 \pm 55.5 \pm 1.48 \pm$

$\begin{array}{llllll}15.8 & 0.5 & 3.4 & 6.5 & 18.3 & 0.06\end{array}$

$70 \pm \quad 14.3^{c} \quad 2.4 \quad 42.4^{d} \pm 55.6 \pm 1.39 \pm$

$\begin{array}{llllll}18.8 & \pm 0.1 & 2.4 & 3 & 11.8 & 0.07\end{array}$

ns e ns e ns ns

$58.1 \pm 13.3^{c} \quad 5.7^{c} \quad 38.7^{c} \pm 54.3 \pm 1.48 \pm$

$\begin{array}{lllll}25.7 & \pm 0.3^{5.7^{c}} & 0.3 & 4.6 & 0.01\end{array}$

${ }^{a}$ Exposure was of respective generations only $(P, F 1)$ or combined $(P+F 1)$. The oocyte size was a histomorphometrical measurement in the ovary, with 5 largest oocytes measured in each of 3 females of each concentration group. There may be bias from absence of sufficient mature oocytes in 2 of 3 females of the 100 $\mathrm{mg} / \mathrm{L}$ group; however, there was still a significant concentration-dependent decrease when tested without this group. Reproduction parameters of $\mathrm{P}$ zebrafish are based on three spawning units per group $\pm S D$, two in the control group (failing reproduction in the third). ${ }^{b}$ Statistically significant concentration dependent change (total eggs: Spearman, $r^{2}=0.3348, P=0.0487$; egg size: $r^{2}$ $=0.4945, P=0.0107)$. In F1 zebrafish, hatching was assessed on 9.9 spawnings average/treatment (range 6-14); there were no data for 100-control.

Malformation was assessed at $16 \mathrm{dph} .^{c} p=0.0145$, Fisher's exact test. For developmental parameters assessed at $42 \mathrm{dph}$, values represent the average $\pm \mathrm{SD}$ of three replicates (two for groups with untreated $\mathrm{P}$ ). ${ }^{c d} p<0.05,0.01$ in paired T-test with appropriate controls (control-control for control-exposed and exposed-control for exposed-exposed). ${ }^{b} p<0.05,0.01$ for concentration dependent linear regression (Spearman); respective $r^{2}$ are 0.3579 and 0.7414 for length and 0.4250 and 0.6887 for weight. 
Environ. Sci. Technol., 40 (1), 74 -81, 2006.

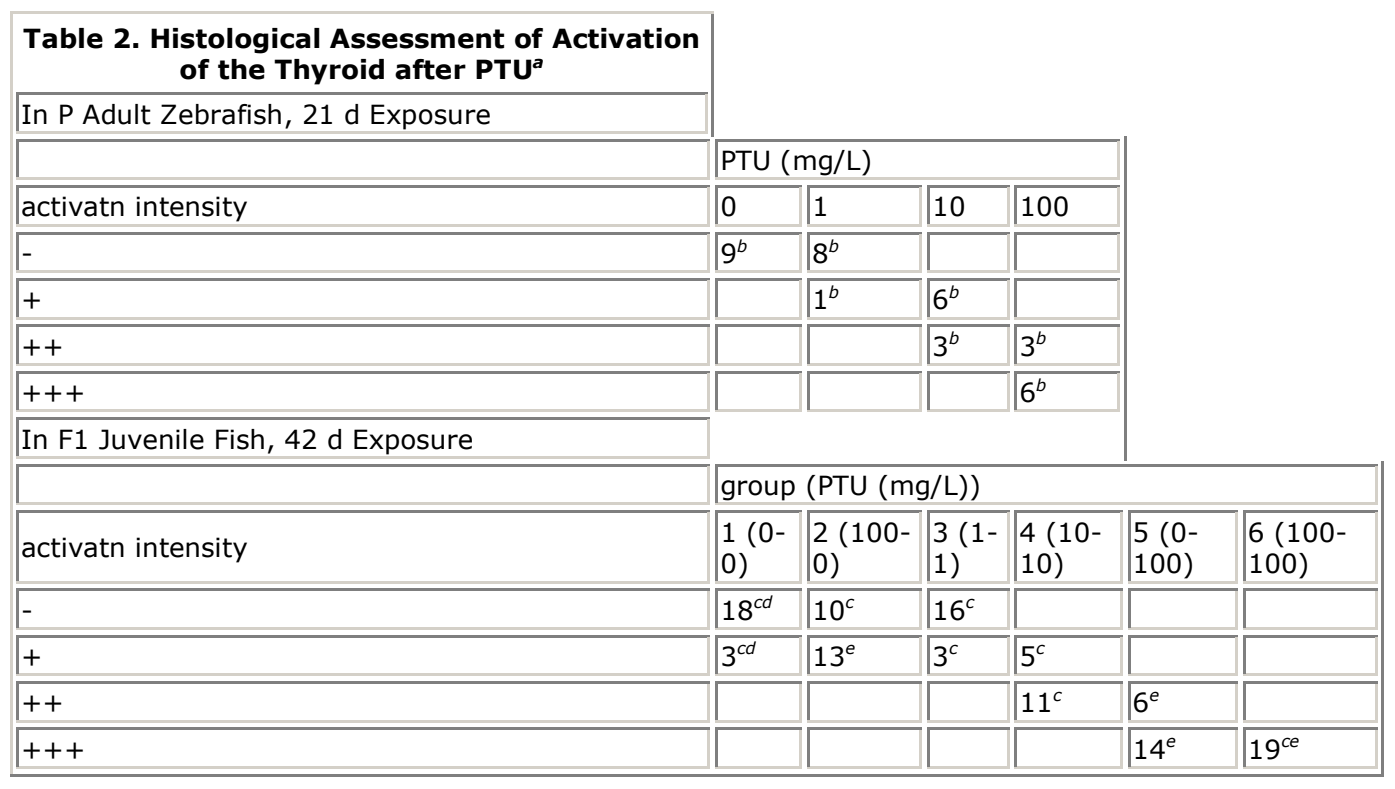

${ }^{a}$ Semiquantitative scoring of descriptors of thyroid follicle epithelial cell synthetic activity (cell height, basophilia, and nucleus hypertrophy). The number of animals/intensity category is given for each exposure concentration. There is a significant dose dependent cell activation in adults. ${ }^{b} \boldsymbol{K}^{2}=55.261$ with $p<0.0001$; $n=9$, exposure 21 days (blind reading); in $\mathrm{F} 1$ juveniles, there is a significant association between activation intensity and PTU concentration for groups with same treatment for $\mathrm{P}$ and $\mathrm{F} 1 .^{c} p<0.0001, K^{2}=132.12$, and also after combining groups with equal juvenile exposure regimes, ignoring parental exposure $(1+2-$ $\left.3-4-5+6, p<0.0001, x^{2}=163.28\right)$. There is an increased activation after parental exposure. ${ }^{d} 0-0$ versus $100-0, p<0.005$. $^{e} 0-100$ versus $100-100, p<$ 0.05 ; Fisher's exact test. In juveniles, the analysis was on thyroids of $16-23$ animals/group, and exposure to PTU was 42 days. 